\title{
A Real-Time Monitoring the TeV Blazars with LHAASO-WCDA detector
}

\author{
Guangman Xiang $^{a b}$, Min Zha ${ }^{b}$, Yi Xing ${ }^{a}$, Jianeng Zhou ${ }^{a}$ for LHAASO collabration \\ ${ }^{a}$ Shanghai Astronomical Observatory, CAS, \\ Shanghai 200030,P.R.China \\ ${ }^{b}$ Institute of High Energy Physics, CAS, \\ Beijing 100049,P.R.China \\ E-mail:gmxiang@ihep.ac.cn
}

\begin{abstract}
With high duty cycle and wide aperture, the Large High Altitude Air Shower Observatory - Water Cherenkov Detector Array(LHAASO-WCDA) can conduct an unbiased gamma-ray sky survey in the energy range from a few hundred $\mathrm{GeV}$ to $100 \mathrm{TeV}$. The sensitivity of WCDA is as high as a few percent of Crab units, which allow us to monitor the VHE variability of blazars. The LHAASO Collaboration has developed an online monitoring program to monitor the extragalactic VHE flare in the WCDA's field of view. Once a flare exceeding the threshold is detected, an alert will be sent to other instruments automatically, and a follow-up multiwavelength observation could be carried out. In this paper, the current status of the monitor, the candidate sources, background estimation method, data reduction, testing running result are presented.
\end{abstract}

36th International Cosmic Ray Conference -ICRC2019-

July 24th - August 1st, 2019

Madison, WI, U.S.A. 


\section{Introduction}

Blazars, including BL Lac and flat-spectrum radio quasars (FSRQ), are Active Galactic Nucleus (AGNs) with relativistic jets viewed closely aligned to our line of sight [1]. In $v-v L(v)$ space, the spectral energy distribution (SED) of blazars presents a two-bump structure. The lowenergy bump ranges from the IR to X-ray energies, which is thought to be originated from the synchrotron radiation of the relativistic electrons accelerated in the magnetic field. The high-energy bump ranges from $\mathrm{MeV}$ to $\mathrm{TeV}$ energies, for which the emission origin is still in debate. In the leptonic scenario, the high-energy emission is originated from the inverse Compton scattering of the low energy photons by the relativistic electrons in the jets, also known as synchrotron self-Compton (SSC) model [2]. In the hadronic scenario, the high-energy emission is attributed to the synchrotron radiation of protons and the $\pi^{0}$ decay process caused by the photon-hadronic interactions [3].

The emission from blazars shows violent variabilities in multiple energy bands from radio to very high energies (VHE; E $>100 \mathrm{GeV}$ ), with timescales range from minutes to years [4]. The luminosity during the flaring states may exceed that during the normal states by two orders of magnitude. This variation usually is considered as the consequence of the intrinsic change of the accretion disk [5]. The study of VHE flares from blazars can help us to understand the VHE emission mechanism during the flares and to distinguish the two emission scenarios. Moreover, observations of blazars during the flares can be used to study the extragalactic background light (EBL) which contains information about the galaxy evolution, to set limits on the Lorentz invariance violation [6], and to research intergalactic magnetic field [7].

Due to the importance of studying flare, many instruments have implemented automatic program to monitor flares, such as ARGO-YBJ [8], HAWC [9]. In this paper, we report how the flare monitor of LHAASO-WCDA works and the future plans of our monitor program.

\section{LHAASO-WCDA experiment}

The Large High Altitude Air Shower Observatory (LHAASO)-water Cherenkov detector ar$\operatorname{ray}(\mathrm{WCDA})$ is located at haizi mountain, Daochen $\left(4410 \mathrm{~m}\right.$ a.s. $1 ., 600 \mathrm{~g} / \mathrm{cm}^{2}, 29^{\circ} 21^{\prime} 31^{\prime \prime} \mathrm{N}, 100^{\circ} 08^{\prime} 15^{\prime \prime}$ E) Sichuan Province, China. It consists of 3 separate pools with a total area of about 78,000 $\mathrm{m}^{2}$. The area of each small pools (No.1 and No.2) is $150 \times 150 \mathrm{~m}^{2}$ with 900 detection units $\left(5 \times 5 \mathrm{~m}^{2}\right)$, and the area of the other pool (No.3) is $300 \times 110 \mathrm{~m}^{2}$ with 1320 detection units. Each detection unit is separated by black plastic curtains to block the scattered light from other units.

With high duty cycle and wide aperture, the WCDA's detection threshold can reach a few percent of $\mathrm{Crab}$ units at $300 \mathrm{GeV}$ to $100 \mathrm{TeV}$ energies in one year survey [10]. This unprecedented sensitivity make WCDA an ideal instrument to conduct online monitoring, early warning and realtime analysis of the extragalactic source. It will provide more samples of the flare event to statistical study the extragalactic source flare phenomenon.

\section{The WCDA Online Flare Monitor}

\subsection{Source selection}

Until now, more than 70 blazars have been observed in VHE band, and most of which are 
HBL(high-energy cutoff BL Lacs) [1]. A total of 62 VHE blazars listed in the TeVCat [11] in the WCDA's field of view are selected as our target sources, as shown in Figure1, and the Crab nebula is selected to supervise the operating status of this program. In addition, we add VHE L3+C [12] to the sources list because a flux enhancement was detected on August 2000. These sources are provided in Table 1 .

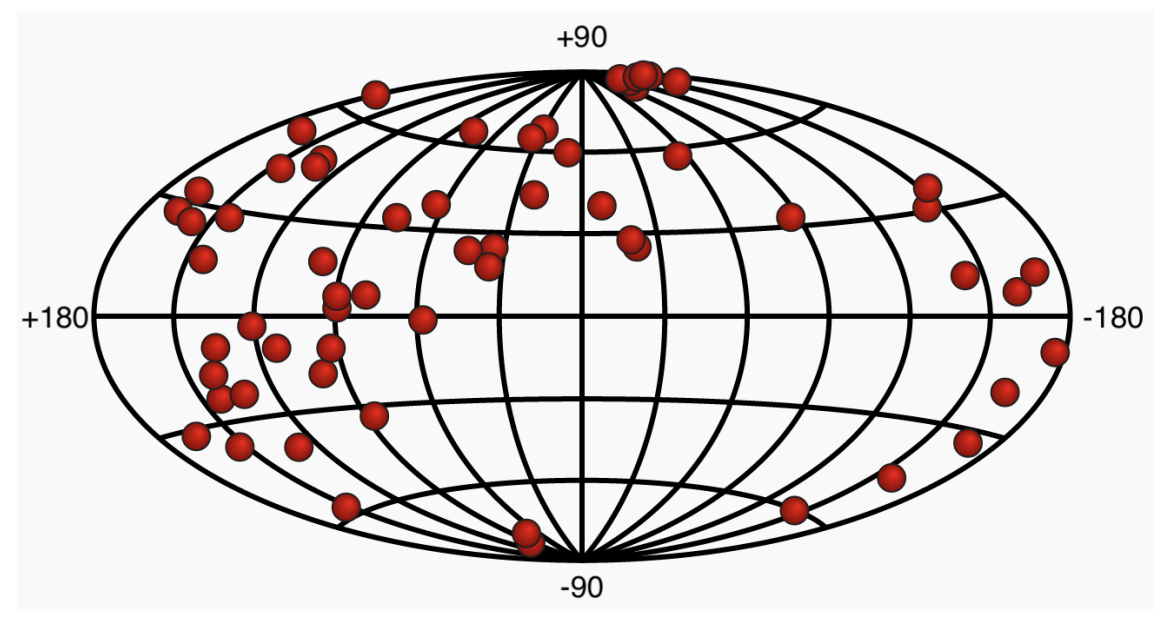

Figure 1: The sky map of selected VHE sources from TeVCat [11] in galactic coordinates.

\subsection{Background estimation}

The surrounding region method is applied to estimate background events. The on-source region is a rectangular cell, and a hollow rectangular cell surrounding it is selected as the off-source region for estimating the background events of the on-source region.Figure 2 shows a schematic diagram of a certain sky area. The hollow off-source region is selected to avoid the influence of the source signal on the background estimation. To ensure that the solid angles of each cell are nearly equal, sky cell widths are $\Delta \delta$ in declination, $\Delta \alpha \simeq \Delta \delta / \cos \delta$ in right ascension.

The width of the sky cell must match the angular resolution of the corresponding $N_{f i t}$ bin. If the width is too small, the signal will be lost too much. On the contrary, if the width is too large, the signal will be submerged in the background. Only the appropriate size of the cell can maximize the signal-to-noise ratio. The value of $\Delta \delta$ should be optimized based on the angular resolution of the detector.As long as the value of the $\Delta \delta$ is determined, the entire division of the sky is determined.

The half-widths of the off-source region are from $\omega_{\alpha}=0.75 \times \Delta \delta$ to $\omega_{\beta}=\Delta \delta+2^{\circ}$ in declination and from $\omega_{\alpha} \times(\Delta \alpha / \Delta \delta)$ to $\omega_{\beta} \times(\Delta \alpha / \Delta \delta)$ in right ascension. The ratio, $R \equiv N_{\text {on }} / N_{\text {off }}$, of the events of on-source region to events from off-source region can be measured by long-term data accumulation, as shown in Figure 3 . The number of background events of on-source region can be calculated by multiplying the number of events from off-source region by the ratio R.

Considering that the sky cells are pre-divided, these sources are probably not located in the center of cell. In order to make the source in the center of the cell, shifts are applied to sky cells both in declination and right ascension. There are a total of 36 shifts, but considering that too many try numbers reduce significance, only the 24 shifts closest to the source are taken to analysis. When the source leaves the field of view, the monitor program analyses the significance of $1,2,4,8$ days. 


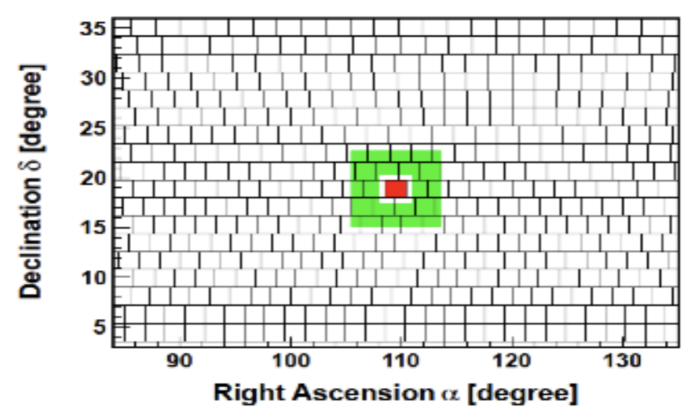

Figure 2: Schematic diagram of the division of the sky. The red cell is a on-source region, the green hollow rectangular cell surrounding it is the corresponding off-source region.

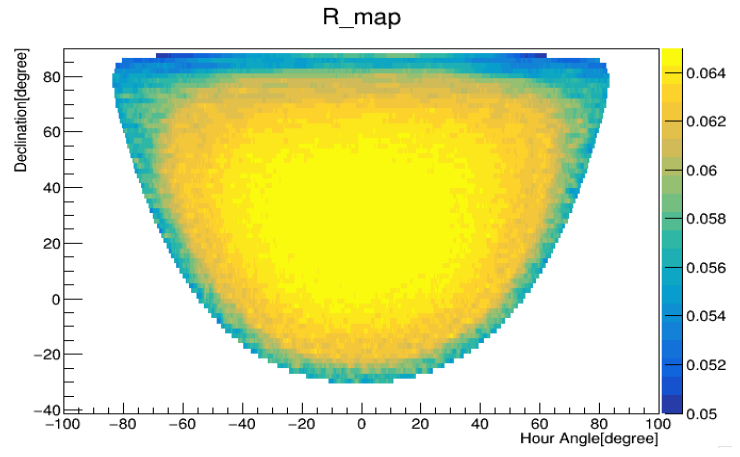

Figure 3: The distribution of ratio R obtained by one month's data.

\subsection{Data reconstruction and selection cuts}

For raw data, the centroid method is applied to reconstruct the shower core position, and the accidental noises are filtered by fitting a narrow planar shower front. Due to the curvature of shower front, the shower direction is obtained by performing a conical correction on the basis of planar fitting. In order to ensure sufficient statistics and good reconstruction quality, the selection cuts we use are as follow:

1.The zenith angle of events should be less than $60^{\circ}$. The particle incident from large $\theta$ pass through thicker atmosphere than that from small $\theta$, so that the maximum of the shower cannot be observed.To keep good reconstruction quality of showers, $\theta \leq 60^{\circ}$ is applied.

2.The number of fired PMTs participating in the conical correction should be more than 20, i.e. $N_{f i t} \geq 20$. $N_{\text {fit }}$ is used to roughly characterize the energy of the event, it is divided into several bins for analysis. The angular resolution of the WCDA No.1 pool is shown in the Figure 4. Each $N_{f i t}$ bin corresponds to an angular resolution, which plays an important role in the division of the sky area in the background estimation. To keep enough statistics, events with $N_{f i t} \geq 20$ are used, which corresponds an angular resolution of $2^{\circ}$.

\subsection{Alarm threshold}

Since the significance of sources are searched from 24 cell shifts and 4 duration shifts, it is difficult to describe the probability of signal excess with a simple distribution. Assuming that there is no signal from source, using the Monte Carlo method to simulate the search process, a significant distribution of statistical fluctuation for a single source can be obtained. The chance probability that a signal's significance greater than a certain threshold can be obtained from simulation result. The thresholds corresponds to chance probability of a source's fake alarms caused by background fluctuations.A detailed simulations are being performed to determine this threshold currently.

\section{Discussion and outlook}

At present, the online flare monitor program is still in the testing phase. Figure 5 shows the monitoring result of Crab since April 9th, 2019.After the program is completed, the daily routine 


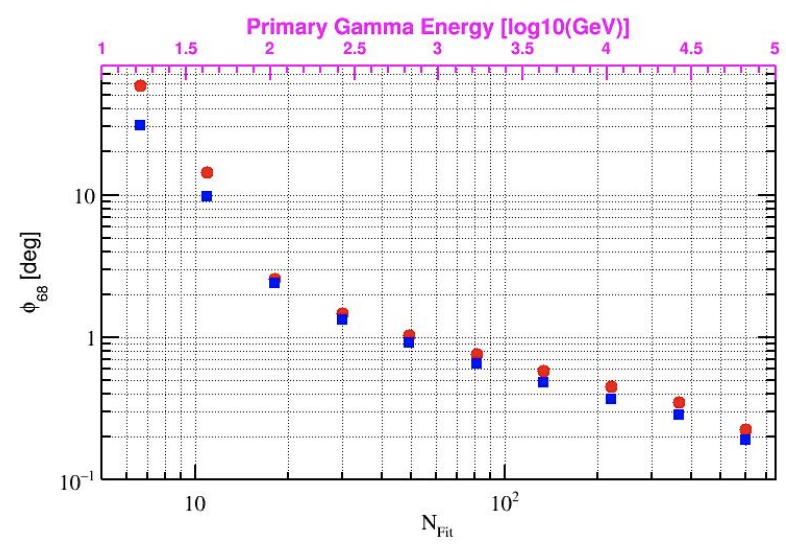

Figure 4: The angular resolution of the different $N_{\text {fit }}$ bins. The red dots and blue dots represent the events whose reconstructed core is located within the array and outside the array respectively. The angular resolution of events that $N_{f i t} \geq 20$ is about $2^{\circ}$

summary will be updated on the website. If a flare exceeding the significance threshold is detected, alert will be sent to the members of the group by e-mail.

The next step is to calculate the alarm threshold through simulation. In addition, $N_{f i t}$ and corresponding $\Delta \delta$ will be further optimized.

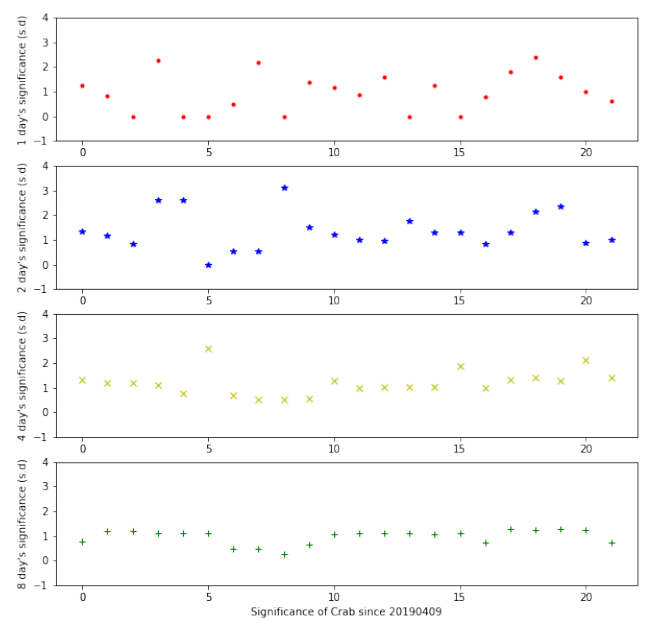

Figure 5: Monitoring results of Crab on different duration shifts since April 9th, 2019. It can be seen from the state of Crab that the current status of the flare monitor is relatively stable.

\section{Acknowledgments}

This work is supported in China by NSFC (11675187), the Chinese Ministry of Science and Technology, the Chinese Academy of Sciences, the Key Laboratory of Particle Astrophysics, IHEP, CAS. 


\section{References}

[1] C. Megan Urry and Paolo Padovani. Unified Schemes for Radio-Loud Active Galactic Nuclei. PASP, 107:803, Sep 1995.

[2] C. D. Dermer, R. Schlickeiser, and A. Mastichiadis. High-energy gamma radiation from extragalactic radio sources. A\&A, 256:L27-L30, Mar 1992.

[3] G. Ghisellini, A. Celotti, G. Fossati, L. Maraschi, and A. Comastri. A theoretical unifying scheme for gamma-ray bright blazars. MNRAS, 301(2):451-468, Dec 1998.

[4] Frank Rieger. Gamma-Ray Astrophysics in the Time Domain. Galaxies, 7(1):28, Jan 2019.

[5] A. Sandrinelli, S. Covino, M. Dotti, and A. Treves. Quasi-periodicities at Year-like Timescales in Blazars. AJ, 151(3):54, Mar 2016.

[6] H. E. S. S. Collaboration, A. Abramowski, F. Acero, F. Aharonian, A. G. Akhperjanian, G. Anton, A. Barnacka, U. Barres de Almeida, A. R. Bazer-Bachi, and Y. Becherini. Search for Lorentz Invariance breaking with a likelihood fit of the PKS 2155-304 flare data taken on MJD 53944. Astroparticle Physics, 34(9):738-747, Apr 2011.

[7] G. Aielli, C. Bacci, F. Barone, B. Bartoli, P. Bernardini, X. J. Bi, C. Bleve, P. Branchini, A. Budano, and S. Bussino. Scaler mode technique for the ARGO-YBJ detector. Astroparticle Physics, 30(2):85-95, Sep 2008.

[8] B. Bartoli, P. Bernardini, X. J. Bi, C. Bleve, I. Bolognino, P. Branchini, A. Budano, A. K. Calabrese Melcarne, P. Camarri, and Z. Cao. Early warning for VHE gamma-ray flares with the ARGO-YBJ detector. Nuclear Instruments and Methods in Physics Research A, 659(1):428-433, Dec 2011.

[9] Thomas Weisgarber, Ian G. Wisher, and for the HAWC Collaboration. Blazar Alerts with the HAWC Online Flare Monitor. arXiv e-prints, page arXiv:1508.05399, Aug 2015.

[10] X. Bai et al. The large high altitude air shower observatory (lhaaso) science white paper, 2019.

[11] Tevcat. http://tevcat.uchicago.edu/.

[12] O. Adriani, M. van den Akker, T. Aziz, J. Bähr, S. Banerjee, F. Becattini, L. Bellucci, B. L. Betev, J. J. Blaising, and G. J. Bobbink. Observation of a VHE cosmic-ray flare-signal with the L3+C muon spectrometer. Astroparticle Physics, 33(1):24-39, Feb 2010. 
Table 1: List of sources selected from TeVCat. The parameters include name, RA(hh,mm,ss),DEC(dd,mm,ss), the threshold of energy, flux, spectrum index, redshift.

\begin{tabular}{|c|c|c|c|c|c|c|}
\hline Name & RA & DEC & $E_{t h}(\mathrm{GeV})$ & FLUX(crab unit) & Index & Redshift \\
\hline OJ 287 & 085449 & +200558 & 150 & 0.013 & 3.49 & 0.3056 \\
\hline TXS 0506+056 & 050925 & +054209 & 110 & 0.016 & 4.8 & 0.3365 \\
\hline S4 0954+65 & 095847 & +653355 & 150 & N/A & N/A & N/A \\
\hline RGB J2056+496 & 205642 & +494007 & 300 & 0.029 & N/A & N/A \\
\hline S3 $0218+35$ & 022105 & +355614 & 100 & 0.3 & 3.8 & 0.954 \\
\hline PKS 0736+017 & 073918 & +013705 & 100 & 0.1 & N/A & 0.18941 \\
\hline TON 0599 & 115931 & +291444 & 100 & 0.3 & N/A & 0.7247 \\
\hline $4 \mathrm{C}+21.35$ & 122454 & +212246 & 100 & 0.03 & 3.75 & 0.432 \\
\hline $3 C 279$ & 125611 & -054722 & 80 & N/A & 4.1 & 0.5362 \\
\hline PKS $1441+25$ & 144356 & +250144 & 80 & 0.16 & N/A & 0.939 \\
\hline PKS 1510-089 & 151252 & -090621 & 150 & 0.03 & 3.26 & 0.361 \\
\hline AP Librae & 151741 & -242219 & 300 & 0.02 & N/A & 0.049 \\
\hline OT 081 & 175132 & +093900 & 150 & 0.08 & N/A & 0.322 \\
\hline S2 $0109+22$ & 011205 & +224439 & 100 & 0.03 & N/A & N/A \\
\hline $3 \mathrm{C} 66 \mathrm{~A}$ & 022241 & +430235 & 200 & 0.06 & 4.1 & 0.34 \\
\hline VER J0521+211 & 052145 & +211251 & 200 & 0.092 & 3.44 & N/A \\
\hline S5 $0716+714$ & 072153 & +712036 & 0.4 & N/A & 3.45 & 0.31 \\
\hline W Comae & 122131 & +281359 & 200 & 0.09 & 200 & 0.102 \\
\hline S3 $1227+25$ & 123014 & +251807 & 100 & 0.07 & N/A & 0.135 \\
\hline MAGIC J2001+435 & 200113 & +435302 & 90 & 0.22 & N/A & N/A \\
\hline BL Lacertae & 220243 & +421640 & 200 & 0.03 & 3.6 & 0.069 \\
\hline 1ES 1101-232 & 110336 & -232945 & 100 & 0.02 & 2.94 & 0.186 \\
\hline 1ES $1218+304$ & 122126 & +301129 & 120 & 0.08 & N/A & 0.182 \\
\hline 1ES $1440+122$ & 144315 & +120011 & 200 & 0.01 & 3.1 & 0.163058 \\
\hline 1ES $1959+650$ & 195959 & +650855 & 600 & 0.64 & N/A & 0.048 \\
\hline H $1426+428$ & 142832 & +424021 & 2500 & 0.03 & 3.5 & 0.129 \\
\hline 1ES $2344+514$ & 234704 & +514249 & 350 & 0.07 & 2.46 & 0.044 \\
\hline Markarian 501 & 165352 & +394537 & 300 & N/A & 2.72 & 0.034 \\
\hline Markarian 421 & 110419 & +381141 & 500 & 0.3 & 2.2 & 0.031 \\
\hline TXS 1515-273 & 151803 & -273134 & N/A & N/A & N/A & N/A \\
\hline TXS $0210+515$ & 021417 & +514451 & 300 & N/A & 1.9 & 0.049 \\
\hline PGC 2402248 & 073326 & +515353 & 200 & 0.01 & N/A & 0.065 \\
\hline 1ES $2037+521$ & 203923 & +521950 & 200 & 0.03 & N/A & 0.053 \\
\hline RGB J2243+203 & 224352 & +201912 & 112 & 0.14 & 4.6 & N/A \\
\hline RX J1136.5+6737 & 113630 & +673704 & 200 & 0.015 & N/A & 0.1342 \\
\hline RBS 0723 & 084712 & +113350 & 200 & 0.025 & N/A & 0.198 \\
\hline H $1722+119$ & 172504 & +115215 & 150 & 0.02 & N/A & N/A \\
\hline MS $1221.8+2452$ & 122424 & +243624 & 200 & 0.04 & N/A & 0.218 \\
\hline RGB J0136+391 & 013632 & +390600 & N/A & N/A & N/A & N/A \\
\hline PKS 0301-243 & 030323 & -240735 & 200 & 0.014 & N/A & 0.2657 \\
\hline KUV 00311-1938 & 003334 & -192133 & N/A & 0.01 & N/A & 0.61 \\
\hline 1ES $0647+250$ & 065046 & +250300 & 100 & 0.03 & N/A & N/A \\
\hline 1ES $1727+502$ & 172818 & +501310 & 150 & 0.021 & N/A & 0.055 \\
\hline
\end{tabular}




\begin{tabular}{crrrrrr}
\hline Name & RA & DEC & $E_{t h}(\mathrm{GeV})$ & FLUX(crab unit) & Index & Redshift \\
\hline 1ES 0033+595 & 003516 & +594724 & 150 & 0.015 & 3.8 & 0.467 \\
1ES 1741+196 & 174401 & +193247 & 180 & 0.016 & 2.7 & 0.084 \\
1ES 1215+303 & 121748 & +300606 & 250 & 0.035 & N/A & 0.131 \\
HESS J1943+213 & 194355 & +211808 & 470 & 0.015 & 3.1 & N/A \\
SHBL J001355.9-185406 & 001352 & -185329 & 310 & 0.006 & 3.4 & 0.095 \\
B3 2247+381 & 225006 & +382558 & 150 & 0.04 & 3.2 & 0.1187 \\
RX J0648.7+1516 & 064845 & +151612 & 200 & 0.033 & 4.4 & 0.179 \\
1ES 0502+675 & 050756 & +673724 & 350 & 0.06 & N/A & 0.34 \\
1ES 0414+009 & 041652 & +010520 & 200 & 0.006 & 3.45 & 0.287 \\
RBS 0413 & 031947 & +184542 & 250 & 0.01 & N/A & 0.19 \\
PKS 1424+240 & 142700 & +234740 & 200 & 0.05 & 4.2 & N/A \\
RGB J0710+591 & 071026 & +590900 & 300 & 0.03 & 2.69 & 0.125 \\
1ES 0806+524 & 080959 & +521900 & 300 & 0.018 & 2.65 & 0.138 \\
RGB J0152+017 & 015233 & +014640 & 300 & 0.02 & 2.95 & 0.08 \\
1ES 1011+496 & 101504 & +492601 & 150 & 0.05 & 3.66 & 0.212 \\
1ES 0347-121 & 034923 & -115838 & 250 & 0.02 & 3.1 & 0.188 \\
1ES 0229+200 & 023253 & +201621 & 580 & 0.018 & 2.5 & 0.1396 \\
Markarian 180 & 113626 & +700927 & 280 & 0.11 & N/A & 0.045 \\
PG 1553+113 & 155544 & +111141 & 300 & 0.034 & 4.5 & 0.5
\end{tabular}

